\title{
Kraft-Pulp Based Material for Electrical Insulation
}

\author{
Rebecca Hollertz, Lars Wågberg* \\ Division of Fibre Technology and ${ }^{*}$ Wallenberg Wood Science Centre, School of Chemical Science \\ and Engineering KTH, Royal Institute of Technology Stockholm, Sweden
}

\author{
Claire Pitois \\ ABB AB Corporate Research, Västerås, Sweden
}

\begin{abstract}
The dielectric properties of the electrical insulation material have a significant influence on the performance and reliability of components in electrical equipment. The influence of the chemistry and electronic structure of the different constituents of the kraft pulp (used in electrical insulation) on some dielectric properties is discussed in this paper. The studies and mechanisms discussed indicate that the presence of different wood polymers (cellulose, hemicellulose and lignin), have different effects on dielectric properties (static electrification and high frequency response). Our results show that the dielectric response of lignin is different compared with the response of hemicellulose and cellulose and this is also expected from the chemical structure of the different components. The lignin molecule has a higher polarizability at frequencies of significance for streamer inception and propagation. With spectroscopic ellipsometry measurements it has also been shown that the energy for electronic transitions in this spectral region is lower for lignin. The results also clearly indicate that the role of cellulose, lignin and hemicellulose should be further investigated for improving electrical breakdown strength of paper based insulation materials.
\end{abstract}

\section{Introduction}

\section{- Electrical insulation from kraft-pulp}

Paper and pressboard from unbleached softwood kraftpulp is used as insulation material in oil-filled highvoltage transformers. In order to improve electrical breakdown strength it is important to aim at limited occurrence and propagation of discharges and streamers. To this end it is essential to have a profound understanding of the fundamental dielectric properties of the cellulosic material; in this paper the influence of the chemistry of the wood fibre components on the dielectric properties are reviewed and discussed. The unbleached kraft-pulp used in electrical insulation contains three main types of biopolymers; cellulose, hemicellulose and lignin typically in the ratio 75:15:3. Cellulose is a polymer with glucose units forming long unbranched chains. The absence of side chains allows cellulose molecules to lie close together and form rigid crystalline structures. Hemicellulose consists of different monosaccharides combined in different ratio and order, with short sidechains connected to the main chain which decrease the structural density compared to cellulose. Softwood lignin consists of mostly of Conferyl and Guiacyl units, which both have one methoxyl group on the phenolic ring. Apart from this, lignin has a branched and complex three-dimensional structure. Unbleached (lignin-containing) pulp is often preferred due to the mechanical strength provided by the remaining hemicellulose and lignin.

\section{Polarizations of wood fibre components}

The dielectric response is used to describe the capacitive behavior of an insulating material in an electric field. The complex permittivity, $\varepsilon$, can be resolved into a real part, storage, $\varepsilon^{\prime}$, and an imaginary component, the loss, $\varepsilon^{\prime \prime}$. With an alternating current (ac) the polarization will result in energy loss of the material as heat. The dielectric loss tangent, $\tan \delta=\varepsilon^{\prime \prime} / \varepsilon^{\prime}$, of wood fibres defines the part of the power applied that is absorbed by the material under the influencing electrical field. A low dielectric loss is desirable for an insulating material. In addition, permittivity differences in composites can lead to the build-up of strong localized electric fields, something that is believed to promote discharges and streamers. Around $50 \mathrm{~Hz}$ the permittivity of mineral oil is between 2.2-2.3, the permittivity of paper is around 5 and oil-impregnated paper hence has a permittivity between that of oil and paper (2.2-5) depending on the paper/oil volume fraction [1].

The response of different basic elements to an alternating electric field is given by their polarizability. The origin and nature of the polarizability depends on the frequency, the higher frequency the shorter is the length scale of polarization. For the inception and propagation of streamers it is also relevant to study fast events, down to the micro and nano second scale. At these high frequencies the only polarizations fast enough to move with electric field is the electronic and atomic polarizations.

\subsection{Electronic polarization}

The electronic polarizability of a molecule can be obtained by summarizing the characteristic polarizabilities, $\alpha_{0}\left(\mathrm{~cm}^{3}\right)=\frac{10^{6}}{4 \pi \varepsilon_{0}} \alpha\left(C \cdot \mathrm{m}^{2} \cdot V^{-1}\right)$, of its covalent bonds. Values for the polarizability of the 
bonds and molecular groups found in the molecular structures of lignin, hemicellulose and cellulose have previously been determined [2].

Hemicellulose and cellulose structures contain aliphatic $\mathrm{C}-\mathrm{C}$ bonds, $\mathrm{C}-\mathrm{H}$ bonds, C-O-C bonds and $\mathrm{C}-\mathrm{O}-\mathrm{H}$ bonds, with electronic polarizabilities between 0.48 and 1.28 (with the unit $4 \pi \varepsilon_{0} \cdot 10^{-30} \mathrm{~m}^{3}$ ). Hemicellulose and cellulose also contain a small amount of carboxylic groups, which contribute to greater polarizability, $\alpha_{0}(\mathrm{C}=\mathrm{O})$ is $1.36\left(4 \pi \varepsilon_{0} \cdot 10^{-30} \mathrm{~m}^{3}\right)$.

Lignin contains aromatic $\mathrm{C}-\mathrm{C}$ bonds, conjugated $\mathrm{C}=\mathrm{C}$ bonds, C-O-C, C-H bonds and $\mathrm{C}-\mathrm{O}-\mathrm{H}$ bonds. The conjugated $\mathrm{C}=\mathrm{C}$ bonds have a higher electronic polarizability, $1.65\left(4 \pi \varepsilon_{0} \cdot 10^{-30} \mathrm{~m}^{3}\right)$, which contributes to the dielectric function in the high frequency range.

\subsection{Atomic polarization}

Atoms bound to each other in molecules do not always share their electrons symmetrically. When an electric field is applied to such atoms or molecules they will be displaced, creating a dipole moment.

\subsection{Dipole polarization}

The macromolecules (cellulose, hemicellulose and lignin), which make up the cell wall in wood, are subjected to dipole polarization. This polarization is due to the displacement of polar groups, relative to the motionless parts of the macromolecules. The loss features of dry cellulose are attributed to the movement of hydroxyl and methyl groups. The structural differences of the Kraft Pulp components will result in different contributions to the dipole polarization.

\subsection{Interfacial polarization}

Polarizable interfaces, contributing to the dielectric losses, are present in heterogeneous dielectrics such as the oil/paper combination where the dielectric constant and conductivity differs in two regions.

\subsection{Low frequency response}

In many dielectrics no loss peak is visible down to the lowest measured frequencies; however, the response rises towards low frequencies. This might be due to DC conductivity which should give a contribution to the imaginary part of the dielectric response. In the low frequency dispersion of many dielectric, such as Kraft paper, both the imaginary and real part rise towards low frequencies which could indicate diffusive conduction due to hopping charges, characterized by a much lower mobility than the free band conduction. Hopping charges are a class of species intermediate between dipoles and charge carriers which spend most of the time in localized states, however, a "hop" to another localized state occurs occasionally when enough energy is available to release the charge out of its potential well. In Kraft paper both the carboxyl groups, which concentration increases with hemicellulose content, as well as the aromatic groups in lignin, could provide localized states. Charges could also be trapped at interfaces, defects or at a bend or kink along a chain.

\section{Ionization and excitation}

The propagation of streamers is powered by a continuous liberation and capture of electrons in the liquid phase. Initiation and propagation of streamers is influenced by electron mobility and by the presence of strong localized electrical fields as well as electron affinity, ionization energy and excitation energy of molecules in the dielectric media. The ionization energy increases with more stable atoms and molecules. The Townsend mechanism describes electron generation by ionization due to electron collisions [3]. The energy of the electron must be at least equal to the ionization energy of the molecule to cause ionization. The ionization energy should therefore be high to avoid increasing the number of electrons generated after collision. Electrons with energy lower than the ionization potential may, however, excite molecules which would then be able to be ionized by electrons with low energy. Photoionization and excitation is also essential in the streamer breakdown mechanism.

It has been shown that additives with low-ionization potential and excitation energies in mineral oil can increase branching of streamers leading to decreased electrical field in the streamer and reduced speed of the propagation at specific voltages [4].

\section{Spectroscopic ellipsometry \\ - High frequency dielectric response of wood fibre components}

Spectroscopic ellipsometry measurements can be used to study the dielectric response and electronic transitions in the ultraviolet-visible-near infrared (UVVIS-NIR) region by measuring polarization changes and calculating the wavelength dependence of the complex refractive index, $\tilde{n}$. In the spectral region $(700-1700 \mathrm{~nm})$ where the extinction coefficient is approximately zero the permittivity $\varepsilon \approx n^{2}$.

In the UV-range the energy for photon absorption, $E_{\mathrm{p}}$ (in $\mathrm{eV}$ ) corresponds to an excitation of an electron from an occupied orbital to an unoccupied or partially unoccupied orbital. The absorption energy can be approximated from the absorption maximum $\omega_{\mathrm{UV}}$ (a spectral parameter which can be calculated from the dielectric response), as $E_{\mathrm{p}} \approx \hbar \omega_{\mathrm{UV}}$ ( $\hbar$ is the reduced Planck constant).

Measurements and calculations of the refractive index and the permittivity of cellulose, glucomannan (hemicellulose) and lignin has shown [5] that lignin has the highest permittivity in the spectral region which is associated with electronic polarization (Figure 1 and Table 1). This can be explained by the presence of conjugated bonds in lignin. 


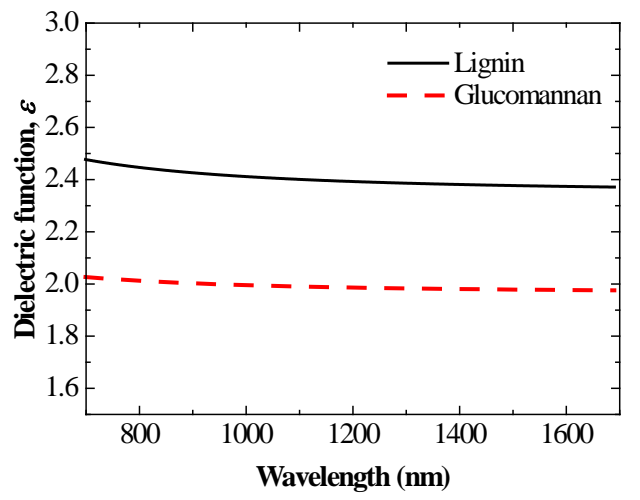

Fig. 1 The dielectric function of glucomannan and lignin approximated from spectroscopic ellipsometry measurements.

Table 1 Approximated wavelengths and energies associated with absorption maxima and permittivity obtained from spectroscopic ellipsometry [5].

\begin{tabular}{l|c|c|c} 
Material & $\varepsilon$ & $\begin{array}{c}\text { Wavelength } \\
\text { (nm) }\end{array}$ & $\begin{array}{c}\text { Photon } \\
\text { energy }(\mathrm{eV})\end{array}$ \\
\hline Lignin & $\sim 2.4$ & 200 & 6.5 \\
\hline $\begin{array}{l}\text { Glucomannan } \\
\text { (Hemicellulose ) }\end{array}$ & $\sim 2.0$ & 165 & 8.0 \\
\hline Cellulose* & $\sim 2.3$ & 146 & 9.1
\end{tabular}

*Calculated from spectral data obtained by Bergström et al. 1999 [7].

The energies of the characteristic absorption frequencies of lignin, glucomannan and cellulose were approximated and are shown in Table 1. Cellulose has, according to this approximation, the highest energy for the main electronic transitions, $9.1 \mathrm{eV}$, whereas it is 8.0 $\mathrm{eV}$ for glucomannan and lignin has a value as low as 6.5 $\mathrm{eV}$. Lignin has, although it is electrically insulating, intriguing electro-chemical properties that has been recognized and e.g. suggested for potential use in energy storage applications [6].

\section{Static electrification at oil/pressboard interfaces}

Static electrification at oil-pressboard interfaces is believed to be the cause of electrical discharges. Paillat et al. have presented a study on the effect of physicochemical properties on static electrification of pressboard [8]. In this study they observed an increased charge accumulation with an increased content of lignin and hemicellulose (however, the difficulty to draw conclusions of the effect of a single parameter was acknowledged, since morphology is also varying with the composition). An increased charge accumulation was also observed for refined pulps and this was explained with a higher availability of surface charges of the refined samples. The charges in kraft-pulp originate mainly from the carboxyl groups in hemicellulose why an increase of the hemicellulose content would, with the same hypothesis, increases the charge of the fibre surfaces. In this earlier work it was also concluded that the lignin-free pulp, which had a higher concentration of crystalline cellulose gave the lowest charge accumulation on the pressboard surface.

\section{Conclusions}

The studies discussed have shown that the composition of the raw materials influence the dielectric properties; where lignin contributes to higher electronic polarizability and lower energy for electronic transitions. Higher electronic polarizability contributes to the build-up of localized E-fields in the case of fast events, relevant for steamer inception and propagation. The lower energy for electronic transition would mean that the lignin molecule is more easily excited or ionized resulting in liberation of electrons which sustain streamers. The kraft-pulp used in electrical insulation contains only a small amount of lignin and its actual influence on the breakdown strength of electrical insulation has to be further investigated to completely understand its consequence. The physicochemical complexity of the pressboard, and the fact that mineral oil similar to lignin contains a large fraction of aromatic compounds and conjugated bonds, further complicates the analysis. In the work described that account for the effect of morphology and chemistry on static electrification it was observed that charge accumulation increase with lignin and hemicellulose content. The increased availability of wood fibre charges, which would also be one off the effects of increased lignin and hemicellulose content, is also concluded to increase the charge accumulation at the interface of oil-pressboard.

\section{References}

[1] R. Hollertz, C. Pitois, and L. Wågberg, "Effect of Composition and Morphology on the Dielectric Response of Cellulose-based Electrical Insulation,” IEEE Trans. Dielectr. Electr. Insul. In press.

[2] Israelaschvili J, Intermolecular and Surface Forces, 2nd ed. San Diego: Academic Press Inc., 1991.

[3] E. Kuffel, W. S. Zaengl, and J. Kuffel, High Voltage Engineering: Fundamentals, 2nd ed. Oxford: Elsevier, 2000.

[4] M. Unge, S. Singha, N. Van Dung, D. Linhjell, S. Ingebrigtsen, and L. E. Lundgaard, "Enhancements in the lightning impulse breakdown characteristics of natural ester dielectric liquids,” Appl. Phys. Lett., vol. 102, no. 17, p. 172905, 2013.

[5] R. Hollertz, H. Arwin, B. Faure, Y. Zhang, L. Bergström, and L. Wågberg, "Dielectric properties of lignin and glucomannan as determined by spectroscopic ellipsometry and Lifshitz estimates of non-retarded Hamaker constants," Cellulose, vol. 20, no. 4, pp. 1639-1648, 2013.

[6] G. Milczarek and O. Inganas, "Renewable Cathode Materials from Biopolymer/Conjugated Polymer 
Interpenetrating Networks,” Science, vol. 335, no. 6075, pp. 1468-1471, 2012.

[7] L. Bergström, S. S. Torbjörn Dahlfors, H. Arwin, and L. Ödberg, "Spectroscopic ellipsometry characterisation and estimation of the Hamaker constant of cellulose," Cellulose, vol. 6, pp. 1-13, 1999.

[8] T. Paillat, L. Onic, O. Moreau, Y. Bertrand, G. Mortha, N. Charvet, and G. Touchard, "Influence of pressboard physico-chemical composition on static electrification in power transformers," IEEE Trans. Ind. Appl., vol. 39, no. 2, pp. 346-354, 2003. 